\title{
Sensitivity curves for searches for gravitational-wave backgrounds
}

\author{
Eric Thrane ${ }^{1, *}$ and Joseph D. Romano ${ }^{2, \dagger}$ \\ ${ }^{1}$ LIGO Laboratory, California Institute of Technology, MS 100-36, Pasadena, California 91125, USA \\ ${ }^{2}$ Department of Physics and Astronomy and Center for Gravitational-Wave Astronomy, \\ University of Texas at Brownsville, Brownsville, Texas 78520, USA
}

(Received 19 October 2013; published 12 December 2013)

\begin{abstract}
We propose a graphical representation of detector sensitivity curves for stochastic gravitational-wave backgrounds that takes into account the increase in sensitivity that comes from integrating over frequency in addition to integrating over time. This method is valid for backgrounds that have a power-law spectrum in the analysis band. We call these graphs "power-law integrated curves." For simplicity, we consider crosscorrelation searches for unpolarized and isotropic stochastic backgrounds using two or more detectors. We apply our method to construct power-law integrated sensitivity curves for second-generation ground-based detectors such as Advanced LIGO, space-based detectors such as LISA and the Big Bang Observer, and timing residuals from a pulsar timing array. The code used to produce these plots is available at https:// dcc.ligo.org/LIGO-P1300115/public for researchers interested in constructing similar sensitivity curves.
\end{abstract}

DOI: 10.1103/PhysRevD.88.124032

PACS numbers: 04.30.-w, 04.80.Nn

\section{INTRODUCTION}

When discussing the feasibility of detecting gravitational waves using current or planned detectors, one often plots characteristic strain $h_{c}(f)$ curves of predicted signals [defined below in Eq. (5)] and compares them to sensitivity curves for different detectors. The sensitivity curves are usually constructed by taking the ratio of the detector's noise power spectral density $P_{n}(f)$ to its sky- and polarization-averaged response to a gravitational wave $\mathcal{R}(f)$, defining $S_{n}(f) \equiv P_{n}(f) / \mathcal{R}(f)$ and an effective characteristic strain noise amplitude $h_{n}(f) \equiv \sqrt{f S_{n}(f)}$. If the curve corresponding to a predicted signal $h_{c}(f)$ lies above the detector sensitivity curve $h_{n}(f)$ in some frequency band, then the signal has signal-to-noise ratio $>1$. An example of such a plot is shown in Fig. 1, which is taken from [1].

For stochastic gravitational waves, which are typically searched for by cross-correlating data from two or more detectors, one often adjusts the height of a sensitivity curve to take into account the total observation time (e.g., $T=1$ yr or 5 yr). For uncorrelated detector noise, the expected (power) signal-to-noise ratio of a crosscorrelation search for a gravitational-wave background for frequencies between $f$ and $f+\delta f$ scales like $\sqrt{T \delta f}$. So the effective characteristic strain noise amplitude $h_{n}(f)$ should be multiplied by a factor of $1 /(T \delta f)^{1 / 4}$. Also, instead of characteristic strain, one often plots the predicted fractional energy density in gravitational waves $\Omega_{\mathrm{gw}}(f)$ as a function of frequency, which is proportional to $f^{2} h_{c}^{2}(f)$ [see Eq. (6)]. An example of such a plot is shown in Fig. 2, which is taken from [2].

But for stochastic gravitational waves, plots such as Figs. 1 and 2 do not always tell the full story. Searches

\footnotetext{
*ethrane@ligo.caltech.edu

†joseph.romano@ligo.org
}

for gravitational-wave backgrounds also benefit from the broadband nature of the signal. The integrated signalto-noise ratio $\rho$ [see Eq. (21)] also scales like $\sqrt{N_{\text {bins }}}=$ $\sqrt{\Delta f / \delta f}$, where $N_{\text {bins }}$ is the number of frequency bins of width $\delta f$ in the total bandwidth $\Delta f$. As we shall see below, the actual value of the proportionality constant depends on the spectral shape of the background and on the detector geometry (e.g., the separation and relative orientation of the detectors), in addition to the individual detector noise power spectral densities. Since this improvement to the sensitivity is signal dependent, it is not always folded into the detector sensitivity curves, even though the improvement in sensitivity can be significant. ${ }^{1}$ And when it is folded in, as in Fig. 2, a single spectral index is assumed, making it difficult to compare published limits with arbitrary models. In other cases, limits are given as a function of spectral index, but the constrained quantity depends on an arbitrary reference frequency; see Eq. (7).

To illustrate the improvement in sensitivity that comes from integrating over frequency, consider the simple case of a white gravitational-wave background signal in white uncorrelated detector noise. In this case, $\rho$ increases by precisely $\sqrt{N_{\text {bins }}}$ compared to the single bin analysis. For ground-based detectors like LIGO, typical values ${ }^{2}$ of $\Delta f$ and $\delta f$ are $\Delta f \approx 100 \mathrm{~Hz}$ and $\delta f \approx 0.25 \mathrm{~Hz}$, leading to $N_{\text {bins }} \approx 400$, and a corresponding improvement in $\rho$ of about 20; see, e.g., [2]. For colored spectra and nontrivial

\footnotetext{
${ }^{1}$ To be clear, integration over frequency is always carried out in searches for stochastic gravitational-wave backgrounds, even though this is not always depicted in sensitivity curves.

${ }^{2}$ The $0.25 \mathrm{~Hz}$ bin width typical of LIGO stochastic analyses is chosen to be sufficiently narrow that one can approximate the signal and noise as constant across the width of the bin, yet sufficiently wide that the noise can be approximated as stationary over the duration of the data segment.
} 


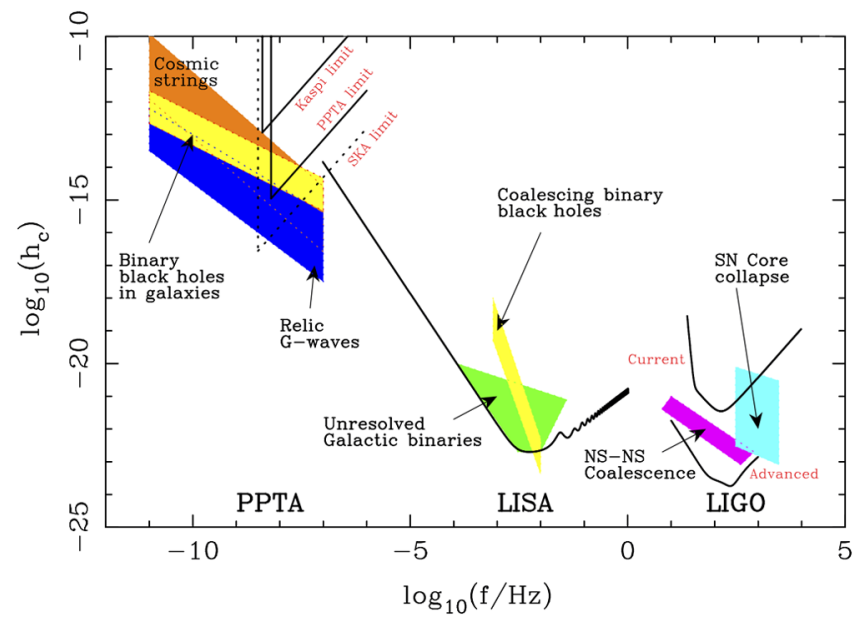

FIG. 1 (color online). Sensitivity curves for gravitational-wave observations and the predicted spectra of various gravitationalwave sources, taken from [1].

detector geometry the improvement will be less, but a factor of $\sim 5-10$ increase in $\rho$ is not unrealistic.

In this paper, we propose a relatively simple way to graphically represent this improvement in sensitivity for gravitational-wave backgrounds that have a power-law frequency dependence in the sensitivity band of the detectors. An example of such a "power-law integrated sensitivity curve" is given in Fig. 3 for a correlation measurement between the Advanced LIGO detectors in Hanford, WA and Livingston, LA. Details of the construction and interpretation of these curves will be given in

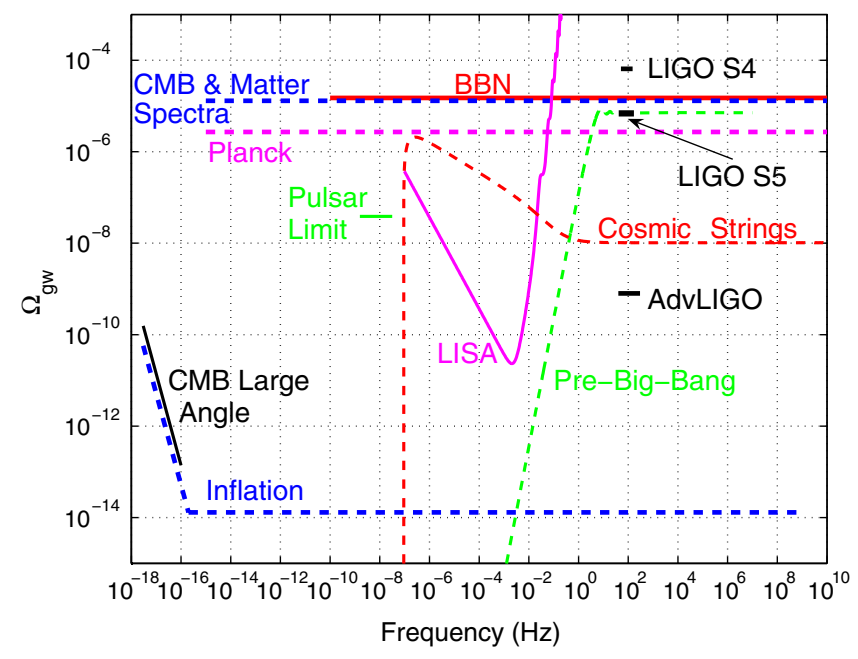

FIG. 2 (color online). Plot showing strengths of predicted gravitational-wave backgrounds in terms of $\Omega_{\mathrm{gw}}(f)$ and the corresponding sensitivity curves for different detectors, taken from [2]. Upper limits from various measurements, e.g., S5 LIGO Hanford-Livingston and pulsar timing, are shown as horizontal lines in the analysis band of each detector. The upper limits take into account integration over frequency, but only for a single spectral index.

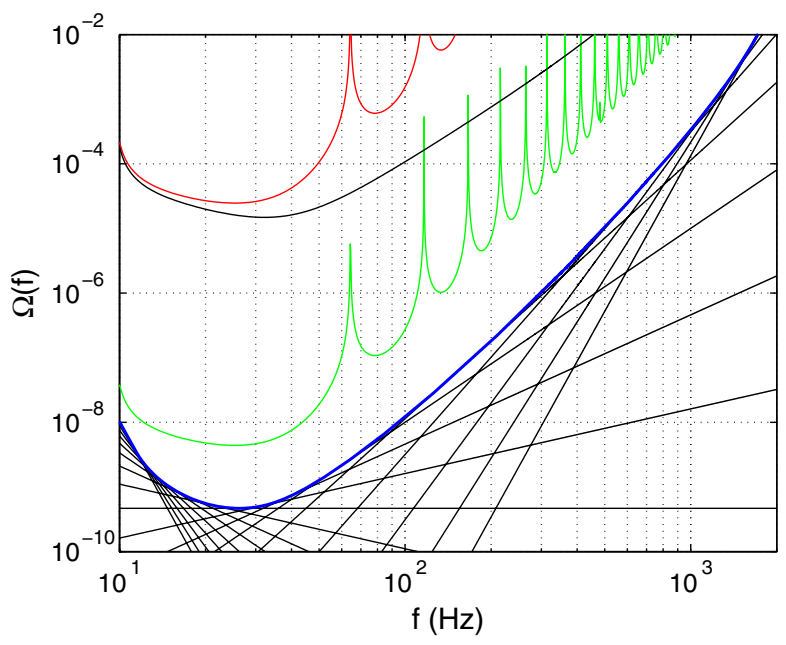

FIG. 3 (color online). $\quad \Omega_{\mathrm{gw}}(f)$ sensitivity curves from different stages in a potential future Advanced LIGO Hanford-LIGO Livingston correlation search for power-law gravitational-wave backgrounds. The top black curve is the single-detector sensitivity curve, assumed to be the same for both $\mathrm{H} 1$ and $\mathrm{L} 1$. The red curve shows the sensitivity of the H1L1 detector pair to a gravitational-wave background, where the spikes are due to zeros in the Hanford-Livingston overlap reduction function (see left panel of Fig. 5). The green curve shows the improvement in sensitivity that comes from integration over an observation time of 1 year for a frequency bin size of $0.25 \mathrm{~Hz}$. The set of black lines is obtained by integrating over frequency for different power-law indices, assuming a signal-to-noise ratio $\rho=1$. Finally, the blue power-law integrated sensitivity curve is the envelope of the black lines. See Sec. III, Fig. 7 for more details.

Sec. III, Fig. 7. We show this figure now for readers who might be anxious to see the results.

In Sec. II we briefly review the fundamentals of crosscorrelation searches for gravitational-wave backgrounds, defining an effective strain noise power spectral density $S_{\text {eff }}(f)$ for a network of detectors. For simplicity, we consider cross-correlation searches for unpolarized and isotropic stochastic backgrounds using two or more detectors. In Sec. III we present a graphical method for constructing sensitivity curves for power-law backgrounds based on the expected signal-to-noise ratio for the search, and we apply our method to construct new power-law integrated sensitivity curves for correlation measurements involving second-generation ground-based detectors such as Advanced LIGO, space-based detectors such as the Big Bang Observer (BBO), and a pulsar timing array. For completeness, we also construct a power-law integrated sensitivity curve for an autocorrelation measurement using LISA. We conclude with a brief discussion in Sec. IV.

\section{FORMALISM}

In this section, we summarize the fundamental properties of a stochastic background and the correlated response 
TABLE I. Summary of select variables with references to key equations.

\begin{tabular}{ll}
\hline \hline Variable & \multicolumn{1}{c}{ Definition } \\
\hline$h_{a b}(t, \vec{x})$ & metric perturbation, Eq. (1) \\
$h_{A}(f, \hat{k})$ & Fourier coefficients of metric perturbation, Eq. (1) \\
$S_{h}(f)$ & strain power spectral density of a gravitational-wave background, Eq. (3) \\
$\Omega_{\mathrm{gw}}(f)$ & fractional energy density spectrum of a gravitational-wave background, Eq. (4) \\
$h_{c}(f)$ & characteristic strain for gravitational waves, Eq. (5) \\
$h(t)$ & detector response to gravitational waves, Eq. (12) \\
$R_{I}^{A}(f, \hat{k})$ & detector response to a sinusoidal plane gravitational wave, Eq. (12) \\
$\tilde{h}(f)$ & Fourier transform of $h(t)$, Eq. (13) \\
$\Gamma_{I J}(f)$ & overlap reduction function for the correlated response to a gravitational-wave background, Eq. $(15)$ \\
$\mathcal{R}_{I}(f)$ & detector response to a gravitational wave averaged over polarizations and directions on the sky, Eq. (17) \\
$P_{h I}(f)$ & detector power spectral density due to gravitational waves, Eq. (18) \\
$P_{n I}(f)$ & detector power spectral density due to noise, Eq. (21) \\
$S_{\text {eff }}(f)$ & effective strain noise power spectral density for a detector network, Eq. $(23)$ \\
$h_{\text {eff }}(f)$ & effective characteristic strain noise amplitude for a detector network, Eq. $(24)$ \\
$S_{n}(f)$ & strain noise power spectral density for a single detector, Eq. $(27)$ \\
$h_{n}(f)$ & characteristic strain noise amplitude for a single detector, $h_{n}(f) \equiv \sqrt{f S_{n}(f)}$ \\
\hline \hline
\end{tabular}

of a network of detectors to such a background. In order to keep track of the many different variables necessary for this discussion, we have included Table I, which summarizes key variables.

\section{A. Statistical properties}

In transverse-traceless coordinates, the metric perturbations $h_{a b}(t, \vec{x})$ corresponding to a gravitational-wave background can be written as a linear superposition of sinusoidal plane gravitational waves with frequency $f$, propagation direction $\hat{k}$, and polarization $A$ :

$h_{a b}(t, \vec{x})=\int_{-\infty}^{\infty} d f \int_{S^{2}} d^{2} \Omega_{\hat{k}} \sum_{A} h_{A}(f, \hat{k}) e_{a b}^{A}(\hat{k}) e^{i 2 \pi f(t-\hat{k} \cdot \vec{x} / c)}$,

where $e_{a b}^{A}(\hat{k})$ are the gravitational-wave polarization tensors and $A=+, \times$ (see, e.g., [3]). The Fourier components $h_{A}(f, \hat{k})$ are random fields whose expectation values define the statistical properties of the background. Without loss of generality we can assume $\left\langle h_{A}(f, \hat{k})\right\rangle=0$. For unpolarized and isotropic stochastic backgrounds, the quadratic expectation values have the form

$\left\langle h_{A}(f, \hat{k}) h_{A^{\prime}}^{*}\left(f^{\prime}, \hat{k}^{\prime}\right)\right\rangle=\frac{1}{16 \pi} \delta\left(f-f^{\prime}\right) \delta_{A A^{\prime}} \delta^{2}\left(\hat{k}, \hat{k}^{\prime}\right) S_{h}(f)$,

where

$$
S_{h}(f)=\frac{3 H_{0}^{2}}{2 \pi^{2}} \frac{\Omega_{\mathrm{gw}}(f)}{f^{3}}
$$

is the gravitational-wave power spectral density, and

$$
\Omega_{\mathrm{gw}}(f)=\frac{1}{\rho_{c}} \frac{d \rho_{\mathrm{gw}}}{d \ln f}
$$

is the fractional contribution of the energy density in gravitational waves to the total energy density needed to close the universe [3]. (Throughout this paper we utilize single-sided power spectra.) The variable $\rho_{c}$ denotes the critical energy density of the universe, while $d \rho_{\mathrm{gw}}$ denotes the energy density between $f$ and $f+d f$. In terms of the characteristic strain defined by

$$
h_{c}(f) \equiv \sqrt{f S_{h}(f)},
$$

it follows that

$$
\Omega_{\mathrm{gw}}(f)=\frac{2 \pi^{2}}{3 H_{0}^{2}} f^{2} h_{c}^{2}(f)
$$

\section{B. Power-law backgrounds}

In this paper, we will restrict our attention to gravitational-wave backgrounds that can be described by power-law spectra:

$$
\Omega_{\mathrm{gw}}(f)=\Omega_{\beta}\left(\frac{f}{f_{\text {ref }}}\right)^{\beta}
$$

where $\beta$ is the spectral index and $f_{\text {ref }}$ is a reference frequency, typically set to $1 \mathrm{yr}^{-1}$ for pulsar timing observations and $100 \mathrm{~Hz}$ for ground-based detectors. The choice of $f_{\text {ref }}$, however, is arbitrary and does not affect the detectability of the signal.

It follows trivially that the characteristic strain also has a power-law form:

$$
h_{c}(f)=A_{\alpha}\left(\frac{f}{f_{\text {ref }}}\right)^{\alpha}
$$


where the amplitude $A_{\alpha}$ and spectral index $\alpha$ are related to $\Omega_{\beta}$ and $\beta$ via

$$
\Omega_{\beta}=\frac{2 \pi^{2}}{3 H_{0}^{2}} f_{\text {ref }}^{2} A_{\alpha}^{2}, \quad \beta=2 \alpha+2 .
$$

For inflationary backgrounds relevant for cosmology, it is often assumed that

$$
\Omega_{\mathrm{gw}}(f)=\mathrm{const},
$$

for which $\beta=0$ and $\alpha=-1$. For a background arising from binary coalescence,

$$
\Omega_{\mathrm{gw}}(f) \propto f^{2 / 3},
$$

for which $\beta=2 / 3$ and $\alpha=-2 / 3$. This power-law dependence is applicable to supermassive black-hole coalescences targeted by pulsar timing observations as well as compact binary coalescences relevant for ground-based and space-based detectors.

\section{Detector response}

The response $h(t)$ of a detector to a passing gravitational wave is the convolution of the metric perturbations $h_{a b}(t, \vec{x})$ with the impulse response $R^{a b}(t, \vec{x})$ :

$$
\begin{aligned}
h(t) & \equiv \int_{-\infty}^{\infty} d \tau \int d^{3} y R^{a b}(\tau, \vec{y}) h_{a b}(t-\tau, \vec{x}-\vec{y}) \\
& =\int_{-\infty}^{\infty} d f \int d^{2} \Omega_{\hat{k}} \sum_{A} R^{A}(f, \hat{k}) h_{A}(f, \hat{k}) e^{i 2 \pi f(t-\hat{k} \cdot \vec{x} / c)},
\end{aligned}
$$

where $\vec{x}$ is the location of the measurement at time $t$. The function $R^{A}(f, \hat{k})$ is the detector response to a sinusoidal plane wave with frequency $f$, propagation direction $\hat{k}$, and polarization $A$. In the frequency domain, we have

$$
\tilde{h}(f)=\int d^{2} \Omega_{\hat{k}} \sum_{A} R^{A}(f, \hat{k}) h_{A}(f, \hat{k}) e^{-i 2 \pi f \hat{k} \cdot \vec{x} / c} .
$$

\section{Overlap reduction function}

Given two detectors, labeled by $I$ and $J$, the expectation value of the cross correlation of the detector responses $\tilde{h}_{I}(f)$ and $\tilde{h}_{J}(f)$ is

$$
\left\langle\tilde{h}_{I}(f) \tilde{h}_{J}^{*}\left(f^{\prime}\right)\right\rangle=\frac{1}{2} \delta\left(f-f^{\prime}\right) \Gamma_{I J}(f) S_{h}(f),
$$

where

$$
\Gamma_{I J}(f) \equiv \frac{1}{8 \pi} \int d^{2} \Omega_{\hat{k}} \sum_{A} R_{I}^{A}(f, \hat{k}) R_{J}^{A *}(f, \hat{k}) e^{-i 2 \pi f \hat{k} \cdot\left(\vec{x}_{I}-\vec{x}_{J}\right) / c}
$$

is the overlap reduction function (see, e.g., [4,5] in the context of ground-based interferometers). Note that $\Gamma_{I J}(f)$ is the transfer function between gravitational-wave strain power $S_{h}(f)$ and detector response cross power $C_{I J}(f)=\Gamma_{I J}(f) S_{h}(f)$. It is often convenient to define a normalized overlap reduction function $\gamma_{I J}(f)$ such that for two identical, colocated and coaligned detectors, $\gamma_{I J}(0)=1$. For identical interferometers with opening angle between the arms $\delta$,

$$
\gamma_{I J}(f)=\left(5 / \sin ^{2} \delta\right) \Gamma_{I J}(f) .
$$

For a single detector (i.e., $I=J$ ), we define

$$
\mathcal{R}_{I}(f) \equiv \Gamma_{I I}(f)
$$

which is the transfer function between gravitational-wave strain power $S_{h}(f)$ and detector response auto power

$$
P_{h I}(f)=\mathcal{R}_{I}(f) S_{h}(f) .
$$

Note that $\mathcal{R}_{I}(f)$ is the antenna pattern of detector $I$ averaged over polarizations and directions on the sky. A plot of $\mathcal{R}_{I}(f)$ normalized to unity for the strain response of an equal-arm Michelson interferometer is shown in Fig. 4.

Detailed derivations and discussions of the overlap reduction functions for ground-based laser interferometers, space-based laser interferometers, and pulsar timing arrays can be found in [3-5], [6,7], and [8,9], respectively. In Fig. 5 we plot the overlap reduction functions for the strain response of the LIGO Hanford-LIGO Livingston detector pair in the long-wavelength limit (valid for frequencies below a few $\mathrm{kHz}$ ) and the strain response of a pair of mini LISA-like Michelson interferometers in the hexagram configuration of the Big Bang Observer (BBO), which is a proposed space-based mission, whose goal is the direct detection of the cosmological gravitational-wave background [10-12]. The two Michelson interferometers for the BBO overlap reduction function are located at opposite

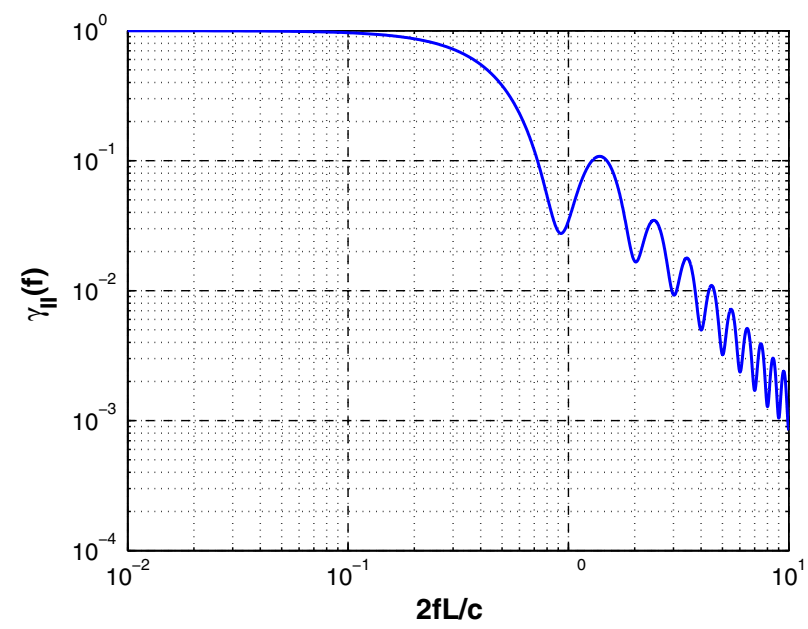

FIG. 4 (color online). A plot of the transfer function $\mathcal{R}_{I}(f)$ normalized to unity for the strain response of an equal-arm Michelson interferometer. The dips in the transfer function occur around integer multiples of $c /(2 L)$, where $L$ is the arm length of the interferometer. 

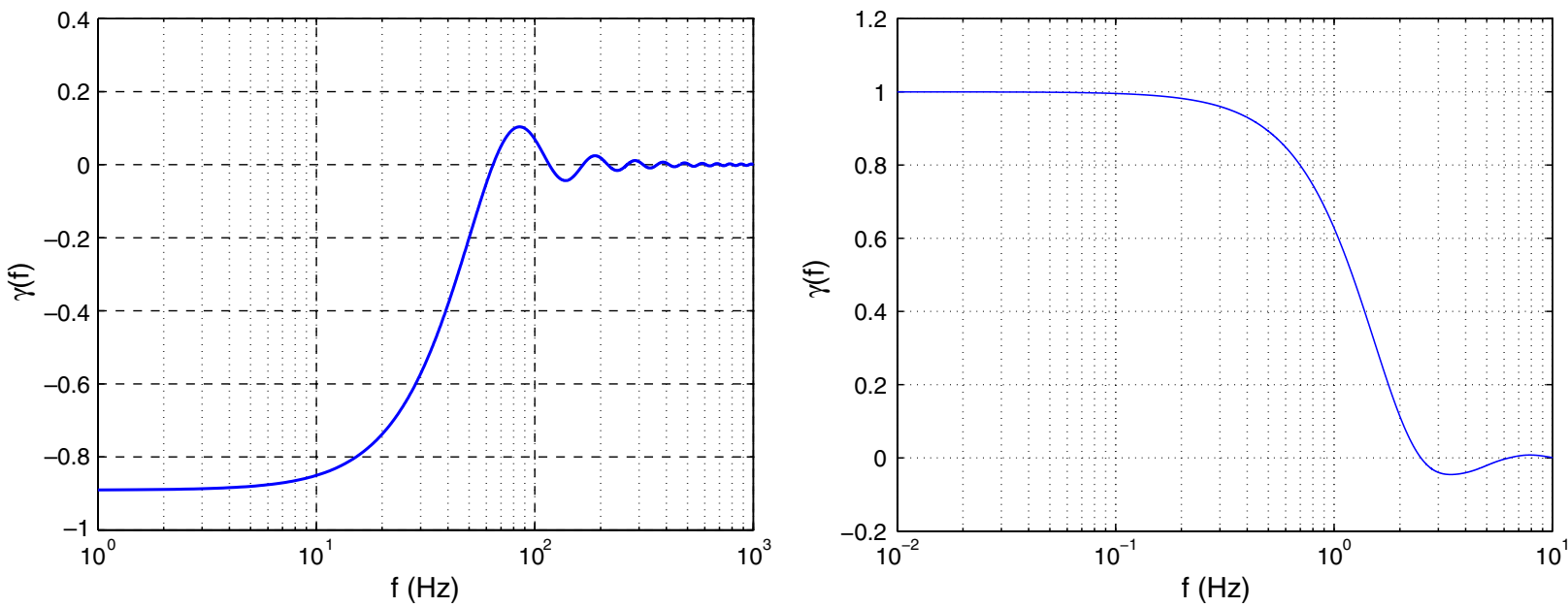

FIG. 5 (color online). Left panel: Normalized overlap reduction function for the LIGO detectors located in Hanford, WA and Livingston, LA. Right panel: Normalized overlap reduction function for two mini LISA-like Michelson interferometers located at opposite vertices of the BBO hexagram configuration.

vertices of a hexagram ("Star of David") and have arm lengths $L=5 \times 10^{7} \mathrm{~m}$ and opening angles $\delta=60^{\circ}$.

In Fig. 6 we plot both the overlap reduction function and the Hellings and Downs curve [8] for the timing response of a pair of pulsars in a pulsar timing array. Assuming two pulsars are separated by an angle $\psi_{I J}$ on the sky, then to a very good approximation [9]

$$
\Gamma_{I J}(f)=\frac{1}{(2 \pi f)^{2}} \frac{1}{3} \zeta_{I J}
$$

where

$$
\begin{gathered}
\zeta_{I J} \equiv \\
\frac{3}{2}\left(\frac{1-\cos \psi_{I J}}{2}\right) \log \left(\frac{1-\cos \psi_{I J}}{2}\right) \\
-\frac{1}{4}\left(\frac{1-\cos \psi_{I J}}{2}\right)+\frac{1}{2}+\frac{1}{2} \delta_{I J}
\end{gathered}
$$

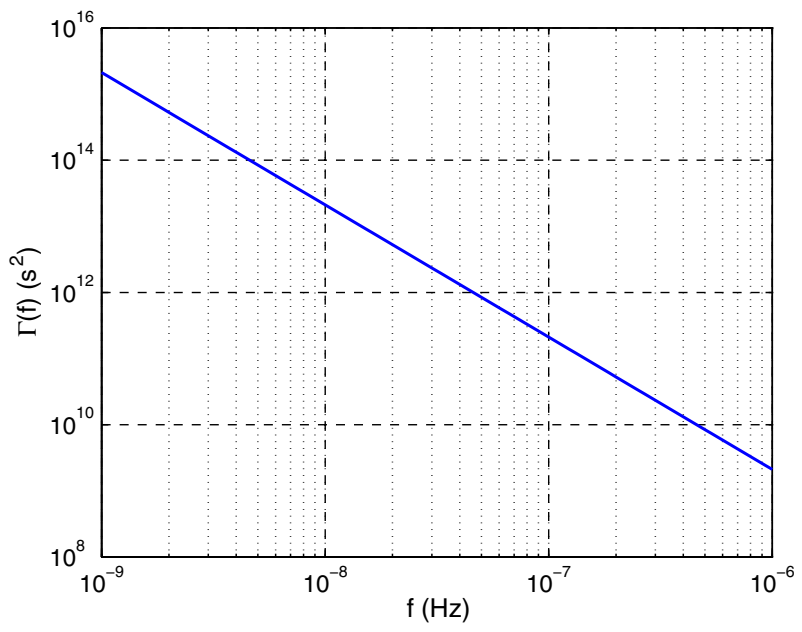

is the Hellings and Downs factor [8]. (The normalization is chosen so that for a single pulsar, $\zeta_{I I}=1$.)

\section{E. Signal-to-noise ratio}

The expected (power) signal-to-noise ratio for a cross-correlation search for an unpolarized and isotropic stochastic background is given by [3]

$$
\rho=\sqrt{2 T}\left[\int_{f_{\min }}^{f_{\max }} d f \frac{\Gamma_{I J}^{2}(f) S_{h}^{2}(f)}{P_{n I}(f) P_{n J}(f)}\right]^{1 / 2},
$$

where $T$ is the total (coincident) observation time and $P_{n I}(f), P_{n J}(f)$ are the auto power spectral densities for the noise in detectors $I, J$. The limits of integration $\left[f_{\min }, f_{\max }\right]$ define the bandwidth of the detector. This is the total broadband signal-to-noise ratio, integrated over both time and frequency. It can be derived as the expected

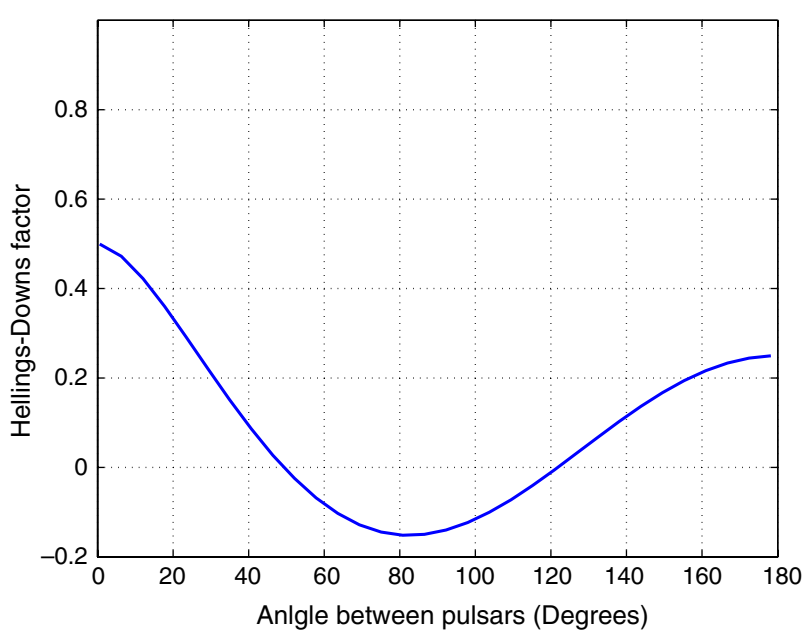

FIG. 6 (color online). Left panel: Overlap reduction function for a pair of pulsars, with $\zeta_{I J}$ chosen to be 0.25 . Right panel: Hellings and Downs function $\zeta\left(\psi_{I J}\right)$. Note that the overlap reduction function is a function of frequency for a fixed pair of pulsars, while the Hellings and Downs function is a function of the angle between two pulsars and is independent of frequency. 
signal-to-noise ratio of a filtered cross correlation of the output of two detectors, where the filter function is chosen so as to maximize the signal-to-noise ratio of the cross correlation. ${ }^{3}$ For a network of detectors, this generalizes to

$$
\rho=\sqrt{2 T}\left[\int_{f_{\min }}^{f_{\max }} d f \sum_{I=1}^{M} \sum_{J>I}^{M} \frac{\Gamma_{I J}^{2}(f) S_{h}^{2}(f)}{P_{n I}(f) P_{n J}(f)}\right]^{1 / 2},
$$

where $M$ is the number of individual detectors, and we have assumed the same coincident observation time $T$ for each detector.

The above expression for $\rho$ suggests the following definition of an effective strain noise power spectral density for the detector network,

$$
S_{\mathrm{eff}}(f) \equiv\left[\sum_{I=1}^{M} \sum_{J>I}^{M} \frac{\Gamma_{I J}^{2}(f)}{P_{n I}(f) P_{n J}(f)}\right]^{-1 / 2},
$$

with corresponding strain noise amplitude

$$
h_{\mathrm{eff}}(f) \equiv \sqrt{f S_{\mathrm{eff}}(f)} .
$$

In terms of $S_{\text {eff }}(f)$, we have

$$
\rho=\sqrt{2 T \delta f} \sqrt{N_{\text {bins }}}\left\langle\frac{S_{h}^{2}}{S_{\text {eff }}^{2}}\right\rangle^{1 / 2},
$$

where \langle\rangle denotes an average ${ }^{4}$ over the total bandwidth of the detectors, $\Delta f=N_{\text {bins }} \delta f$. For the case of $M$ identical, colocated and coaligned detectors, things simplify further. First,

$$
S_{\mathrm{eff}}(f)=\sqrt{\frac{2}{M(M-1)}} S_{n}(f)
$$

where

$$
S_{n}(f) \equiv P_{n}(f) / \mathcal{R}(f)
$$

is the strain noise power spectral density in a single detector. Second,

$$
\rho=\sqrt{T \delta f} \sqrt{N_{\text {bins }}} \sqrt{M(M-1)}\left\langle\frac{S_{h}^{2}}{S_{n}^{2}}\right\rangle^{1 / 2} .
$$

Thus, we see that the expected signal-to-noise ratio scales linearly with the number of detectors for $M \gg 1$, the square root of the total observation time, and the square root of the number of frequency bins. Note that $\sqrt{T \delta f} \sqrt{N_{\text {bins }}}=\sqrt{T \Delta f}$, which is the total time-frequency volume of the measurement.

\footnotetext{
${ }^{3}$ The above expression for $\rho$ assumes that the gravitationalwave background is weak compared to the instrumental noise in the sense that $P_{h I}(f) \ll P_{n I}(f)$ for all frequencies in the bandwidth of the detectors.

${ }^{4}$ Explicitly, $\langle X\rangle \equiv(1 / \Delta f) \int_{f_{\min }}^{f_{\max }} X(f) d f$.
}

\section{POWER-LAW INTEGRATED CURVES}

\section{A. Construction}

The sensitivity curves that we propose are based on Eq. (22) for the expected signal-to-noise ratio $\rho$, applied to gravitational-wave backgrounds with power-law spectra. These "power-law integrated sensitivity curves" include the improvement in sensitivity that comes from the broadband nature of the signal, via the integration over frequency. The following construction is cast in terms of $\Omega_{\mathrm{gw}}(f)$, but we note that power-law integrated curves can also easily be constructed for $h_{c}(f)$ or $S_{h}(f)$ using Eqs. (3) and (5) to convert between the different quantities.

(1) Begin with the detector noise power spectral densities $P_{n I}(f), P_{n J}(f)$, and the overlap reduction functions $\Gamma_{I J}(f)$ for two or more detectors. Using Eq. (23), first calculate the effective strain power spectral density $S_{\text {eff }}(f)$ and then convert it to energy density units $\Omega_{\text {eff }}(f)$ using Eq. (3).

(2) Assume an observation time $T$, typically between 1 and $10 \mathrm{yr}$.

(3) For a set of power-law indices, e.g., $\beta=$ $\{-8,-7, \ldots 7,8\}$, and some choice of reference frequency $f_{\text {ref }}$, calculate the value of the amplitude $\Omega_{\beta}$ such that the integrated signal-to-noise ratio has some fixed value, e.g., $\rho=1$. Explicitly,

$$
\Omega_{\beta}=\frac{\rho}{\sqrt{2 T}}\left[\int_{f_{\min }}^{f_{\max }} d f \frac{\left(f / f_{\mathrm{ref}}\right)^{2 \beta}}{\Omega_{\mathrm{eff}}^{2}(f)}\right]^{-1 / 2} .
$$

Note that the choice of $f_{\text {ref }}$ is arbitrary and will not affect the sensitivity curve.

(4) For each pair of values for $\beta$ and $\Omega_{\beta}$, plot $\Omega_{\mathrm{gw}}(f)=$ $\Omega_{\beta}\left(f / f_{\text {ref }}\right)^{\beta}$ versus $f$.

The envelope of the $\Omega_{\mathrm{gw}}(f)$ power-law curves is the power-law integrated sensitivity curve for a correlation measurement using two or more detectors. Formally, the power-law integrated curve is given by

$$
\Omega_{\mathrm{PI}}(f)=\max _{\beta}\left[\Omega_{\beta}\left(\frac{f}{f_{\text {ref }}}\right)^{\beta}\right] .
$$

Interpretation: Any line (on a log-log plot) that is tangent to the power-law integrated sensitivity curve corresponds to a gravitational-wave background power-law spectrum with an integrated signal-to-noise ratio $\rho=1$. This means that if the curve for a predicted background lies everywhere below the sensitivity curve, then $\rho<1$ for such a background. On the other hand, if the curve for a predicted power-law background with spectral index $\beta$ lies somewhere above the sensitivity curve, then it will be observed with an expected value of $\rho=\Omega_{\beta}^{\text {pred }} / \Omega_{\beta}>1$. Graphically, $\Omega_{\beta}^{\text {pred }}$ is the value of the predicted power-law spectrum evaluated at $f_{\text {ref }}$, while $\Omega_{\beta}$ is the value of the 
same power-law spectrum that is tangent to the sensitivity curve, also evaluated at $f_{\text {ref }}$.

\section{B. Plots}

The calculation of a power-law integrated sensitivity curve is demonstrated in the left-hand panel of Fig. 7 for the Hanford-Livingston (H1L1) pair of Advanced LIGO detectors. Following steps $1-5$ above, we begin with the design detector noise power spectral density $P_{n}(f)$ for an Advanced LIGO detector [13] (which we assume to be the same for both $\mathrm{H} 1$ and L1) and divide by the absolute value of the H1L1 overlap reduction function to obtain the effective strain spectral density $S_{\text {eff }}(f)=$ $P_{n}(f) /\left|\Gamma_{\mathrm{H} 1 \mathrm{~L} 1}(f)\right|$ of the detector pair to a gravitationalwave background [see Eq. (23)]. We then convert $S_{\text {eff }}(f)$ to an energy density $\Omega_{\text {eff }}(f)$ via Eq. (3) to obtain the solid red curve. After integrating $1 \mathrm{yr}$ of coincident data and assuming a frequency bin width of $0.25 \mathrm{~Hz}$, we obtain the solid green curve, which is lower by a factor of $1 / \sqrt{2 T \delta f}$. (The green curve, which depends on the somewhat arbitrary value of $\delta f$, can be thought of as an intermediate data product in LIGO analyses.) Then assuming different spectral indices $\beta$, we integrate over frequency [see Eq. (29)], setting $\rho=1$ to determine the amplitude $\Omega_{\beta}$ of a power-law background. This gives us the set of black lines for each power-law index $\beta$. The blue power-law integrated curve is the envelope of these black lines.

The right-hand panel of Fig. 7 illustrates how to interpret a power-law integrated sensitivity curve. We replot the green and blue curves from the left-hand panel, which respectively represent the time-integrated and power-law integrated sensitivity of an Advanced LIGO H1L1 correlation measurement to a gravitational-wave background. Additionally, we plot two theoretical spectra of the form $\Omega_{\mathrm{gw}}(f) \propto f^{2 / 3}$, which is expected for a background due to compact binary coalescences. The dark brown line corresponds to a somewhat pessimistic scenario in which Advanced LIGO, running at design sensitivity, would detect $\approx 10$ individual binary-neutron-star coalescences per year of science data [14]. The light brown line represents a somewhat optimistic model in which Advanced LIGO, running at design sensitivity, would detect $\approx 100$ individual binary-neutron-star coalescences per year of science data [14]. (A binary-neutron-star detection rate of $40 \mathrm{yr}^{-1}$ is considered a realistic rate for Advanced LIGO [15].)
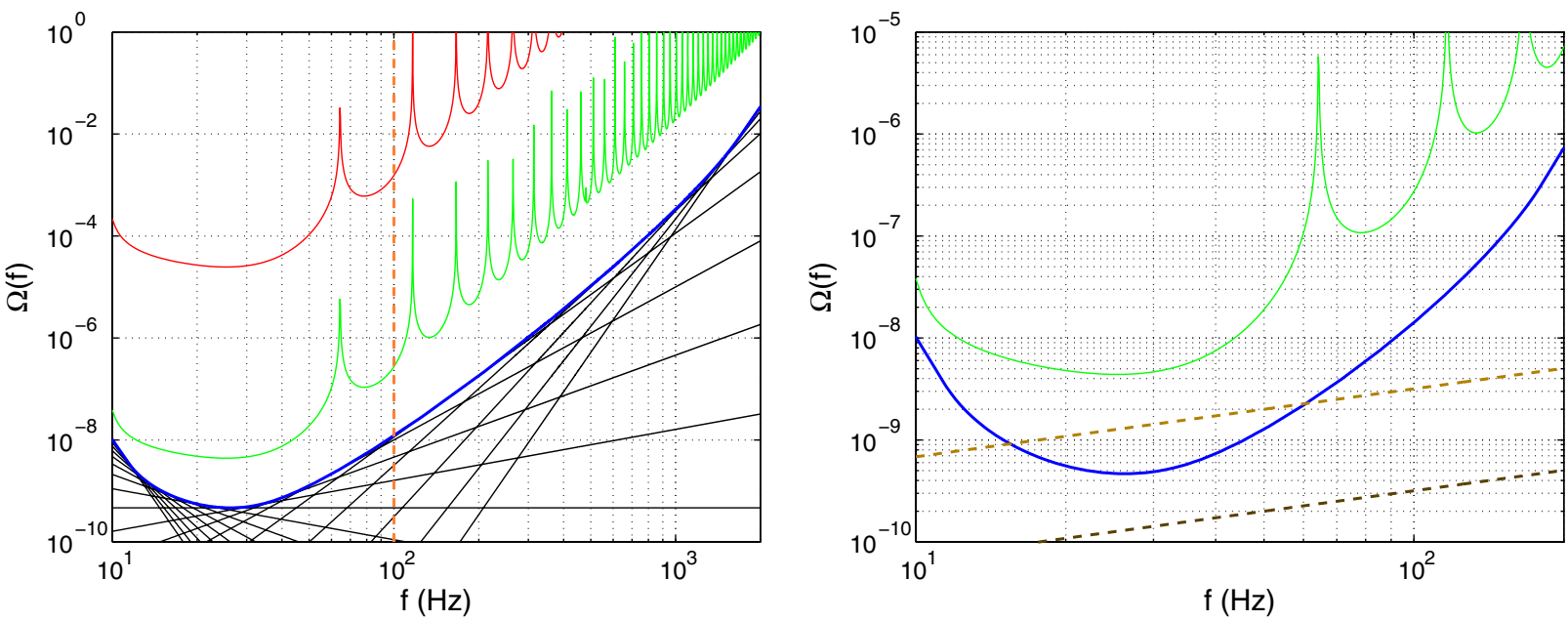

FIG. 7 (color online). Left panel: $\Omega_{\mathrm{gw}}(f)$ sensitivity curves from different stages in a potential future Advanced LIGO H1L1 correlation search for power-law gravitational-wave backgrounds. (For readers of a grayscale copy, we are starting at the top of the plot and working toward the bottom.) The red line shows the effective strain spectral density $S_{\text {eff }}(f)=P_{n}(f) /\left|\Gamma_{\text {H1L1 }}(f)\right|$ of the H1L1 detector pair to a gravitational-wave background signal converted to energy density $\Omega_{\text {eff }}(f)$ via Eq. (3). [The $P_{n}(f)$ used in this calculation is the design detector noise power spectral density for an Advanced LIGO detector, assumed to be the same for both H1 and L1.] The spikes in the red curve are due to zeros in the overlap reduction function $\Gamma_{\mathrm{H} 1 \mathrm{~L} 1}(f)$, which is shown in the left panel of Fig. 5. The green curve, $S_{\text {eff }}(f) / \sqrt{2 T \delta f}$, is obtained through the optimal combination of $1 \mathrm{yr}$ of data, assuming a frequency bin width of $0.25 \mathrm{~Hz}$, as is typical [2]. The vertical dashed orange line marks a typical Advanced LIGO reference frequency, $f_{\text {ref }}=100 \mathrm{~Hz}$. The set of straight black lines is obtained by performing the integration in Eq. (29) for different power-law indices $\beta$, requiring that $\rho=1$ to determine $\Omega_{\beta}$. Finally, the blue power-law integrated sensitivity curve is the envelope of the black lines. Right panel: A demonstration of how to interpret a powerlaw integrated curve. The thin green line and thick blue line are the same as in the left panel. The two dashed brown lines represent two different plausible signal models for gravitational-wave backgrounds arising from binary-neutron-star coalescence; see, e.g., [14]. In each case, $\Omega_{\mathrm{gw}}(f) \propto f^{2 / 3}$; however, the two curves differ by an order of magnitude in the overall normalization of $\Omega_{\mathrm{gw}}(f)$. The louder signal will induce a signal-to-noise ratio $\rho>1$ with an Advanced LIGO H1L1 correlation measurement as it intersects the blue power-law integrated curve-even though it falls below the time-integrated green curve. The weaker signal will induce a signal-to-noise ratio $\rho<1$ with Advanced LIGO H1L1, as it is everywhere below the power-law integrated curve. 

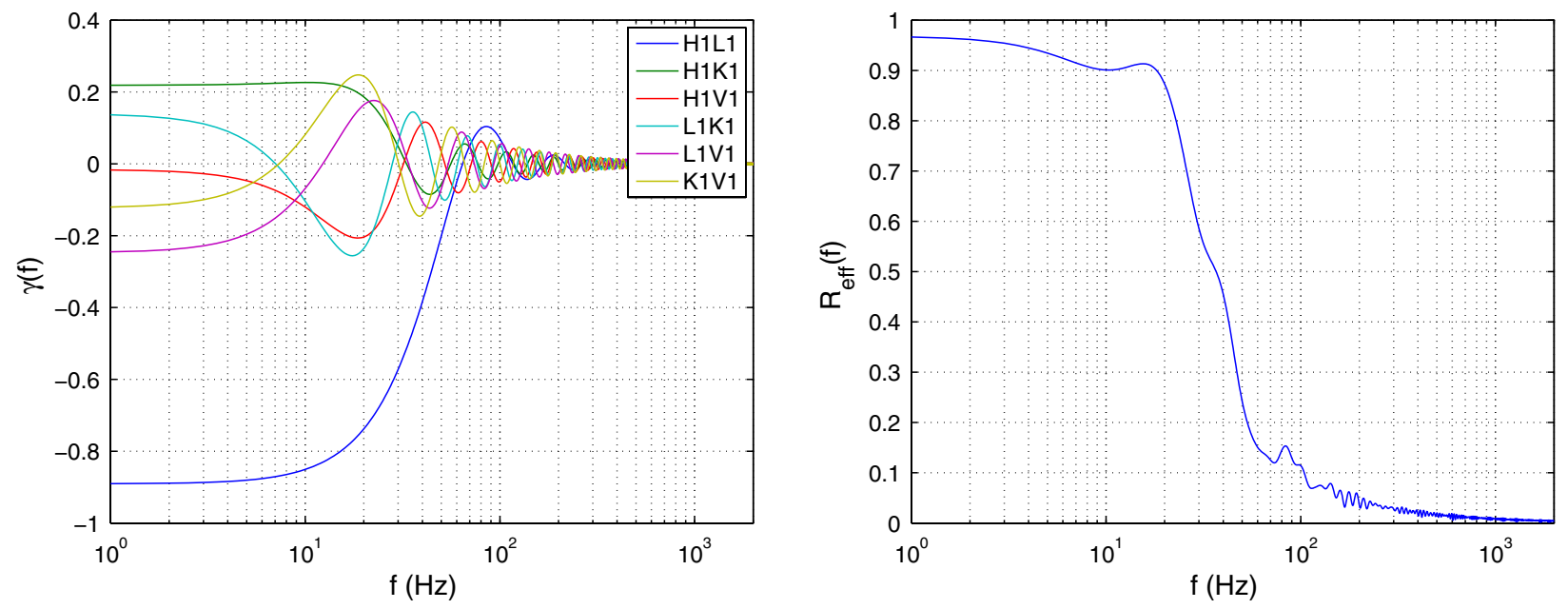

FIG. 8 (color online). Left panel: Individual normalized overlap reduction functions for the six different detector pairs comprising the H1L1K1V1 network. Right panel: Sky- and polarization-averaged response of the H1L1V1K1 network to a gravitational-wave background.

The light-brown curve intersects the blue power-law integrated curve, indicating that the somewhat optimistic model will induce a signal-to-noise ratio $\rho>1$. The dark brown curve falls below the blue power-law integrated curve, indicating that the somewhat pessimistic model will induce a signal-to-noise ratio $\rho<1$. Note that neither curve intersects the green time-integrated sensitivity curve.

In the following subsections, we plot power-law integrated sensitivity curves for several upcoming or proposed experiments: networks of Advanced LIGO detectors (Fig. 9), BBO (Fig. 10, top panel), LISA (Fig. 10, middle panel), and a network of pulsars from a pulsar timing array (Fig. 10, bottom panel).

\section{Advanced LIGO networks}

For the Advanced LIGO networks, we use the design detector noise power spectral density $P_{n}(f)$ taken from [13], assumed to be the same for every detector in the network. We consider three networks: H1L1 (just the US aLIGO detectors), $\mathrm{H} 1 \mathrm{H} 2$ (a hypothetical colocated pair of aLIGO detectors), and H1L1V1K1 (the US aLIGO detectors plus detector pairs created with Virgo V1 and KAGRA K1). ${ }^{5}$ In reality, Virgo and KAGRA are expected to have different noise curves than aLIGO, but we assume the same aLIGO noise for each detector in order to show how the sensitivity curve changes by adding additional identical detectors to the network. Given this assumption, the effective strain power spectral density can be written as

$$
S_{\text {eff }}(f)=P_{n}(f) / \mathcal{R}_{\text {eff }}(f)
$$

\footnotetext{
${ }^{5}$ We have taken the location and orientation of the KAGRA detector to be that of the TAMA 300-m interferometer in Tokyo, Japan. We have not included the planned LIGO India detector [16] in this network, as the precise LIGO-India site has not yet been decided upon.
}

where

$$
\mathcal{R}_{\mathrm{eff}}(f)=\left[\sum_{I=1}^{M} \sum_{J>I}^{M} \Gamma_{I J}^{2}(f)\right]^{1 / 2}
$$

is the sky- and polarization-averaged response of the network to a gravitational-wave background. A plot of the various overlap reduction functions $\gamma_{I J}(f)$ and $\mathcal{R}_{\text {eff }}(f)$ for the H1L1V1K1 network is given in Fig. 8. The resulting power-law integrated sensitivity curves are shown in Fig. 9.

\section{Big bang observer}

For the BBO sensitivity curve, the noise power spectral density for the two Michelson interferometers is taken to be

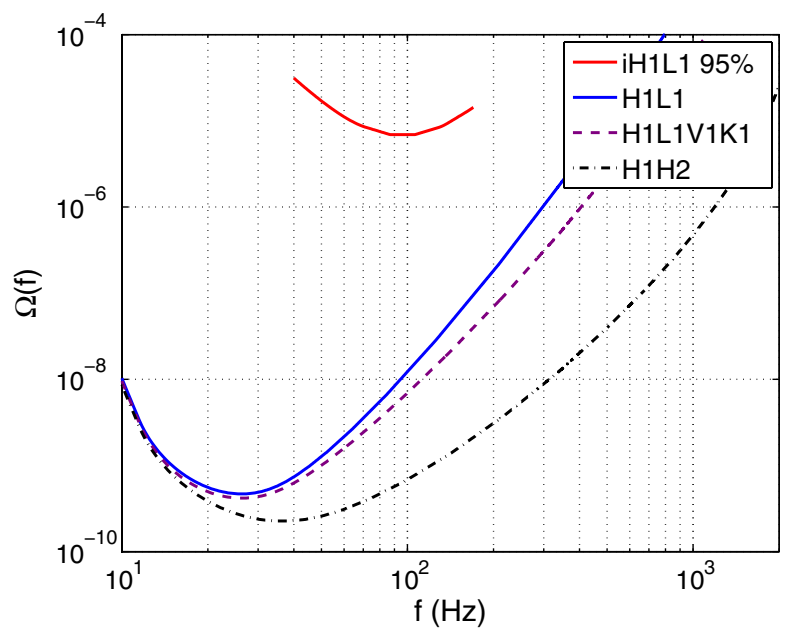

FIG. 9 (color online). Different networks of advanced detectors assuming $T=1 \mathrm{yr}$ of observation. We also include $95 \% \mathrm{CL}$ limits from initial LIGO for comparison [2]. 


$$
P_{n}(f)=\frac{4}{L^{2}}\left[(\widetilde{\delta x})^{2}+\frac{(\widetilde{\delta a})^{2}}{(2 \pi f)^{4}}\right]
$$

where

$$
\begin{gathered}
(\widetilde{\delta x})^{2}=2 \times 10^{-34} \frac{\mathrm{m}^{2}}{\mathrm{~Hz}}, \\
(\widetilde{\delta a})^{2}=9 \times 10^{-34} \frac{\mathrm{m}^{2}}{\mathrm{~s}^{4} \cdot \mathrm{Hz}}
\end{gathered}
$$

are the position and acceleration noise (see Table II from [11]) and $L=5 \times 10^{7} \mathrm{~m}$ is the arm length. Following [12], we have included an extra factor of 4 multiplying the first term in Eq. (33), which corresponds to highfrequency noise that is 4 times larger than shot noise alone. The overlap reduction function for the Michelson interferometers located at opposite vertices of the BBO hexagram is shown in the right panel of Fig. 5. The power-law integrated curve for BBO is given in Fig. 10, top panel.

\section{LISA}

For LISA, the analysis is necessarily different since the standard cross-correlation technique used for multiple detectors such as an Advanced LIGO network, BBO, or a pulsar timing array is not possible for a single LISA constellation. This is because the two independent Michelson interferometers that one can synthesize from the six links of the standard equilateral LISA configuration are rotated at $45^{\circ}$ with respect to one another, leading to zero cross correlation for an isotropic gravitational-wave background for frequencies below about $c / 2 L=3 \times 10^{-2} \mathrm{~Hz}$ [17]. It is possible, however, to construct a combination of the LISA data whose response to gravitational waves is highly suppressed at these frequencies and hence can be used as a real-time noise monitor for LISA $[18,19]$. It is also possible to exploit the differences between the transfer function and spectral shape of a gravitationalwave background and that due to instrumental noise and/or an astrophysical foreground (e.g., from galactic white-dwarf binaries) to discriminate a gravitational-wave background from these other noise contributions [20,21].

For the ideal case of an autocorrelation measurement in a single detector assuming perfect subtraction of instrumental noise and/or any unwanted astrophysical foreground, Eq. (21) for the expected signal-to-noise ratio is replaced by

$$
\rho=\sqrt{T}\left[\int_{0}^{\infty} d f \frac{\mathcal{R}^{2}(f) S_{h}^{2}(f)}{P_{n}^{2}(f)}\right]^{1 / 2},
$$

where $\mathcal{R}(f) \equiv \Gamma(f)$ is the transfer function of the detector and $P_{n}(f)$ is its noise power spectral density. (The $\sqrt{2}$ reduction in $\rho$ compared to a cross-correlation analysis is due to the use of data from only one detector instead of two.) For standard LISA,

$$
P_{n}(f)=\frac{1}{L^{2}}\left[(\widetilde{\delta x})^{2}+\frac{4(\widetilde{\delta a})^{2}}{(2 \pi f)^{4}}\right]
$$
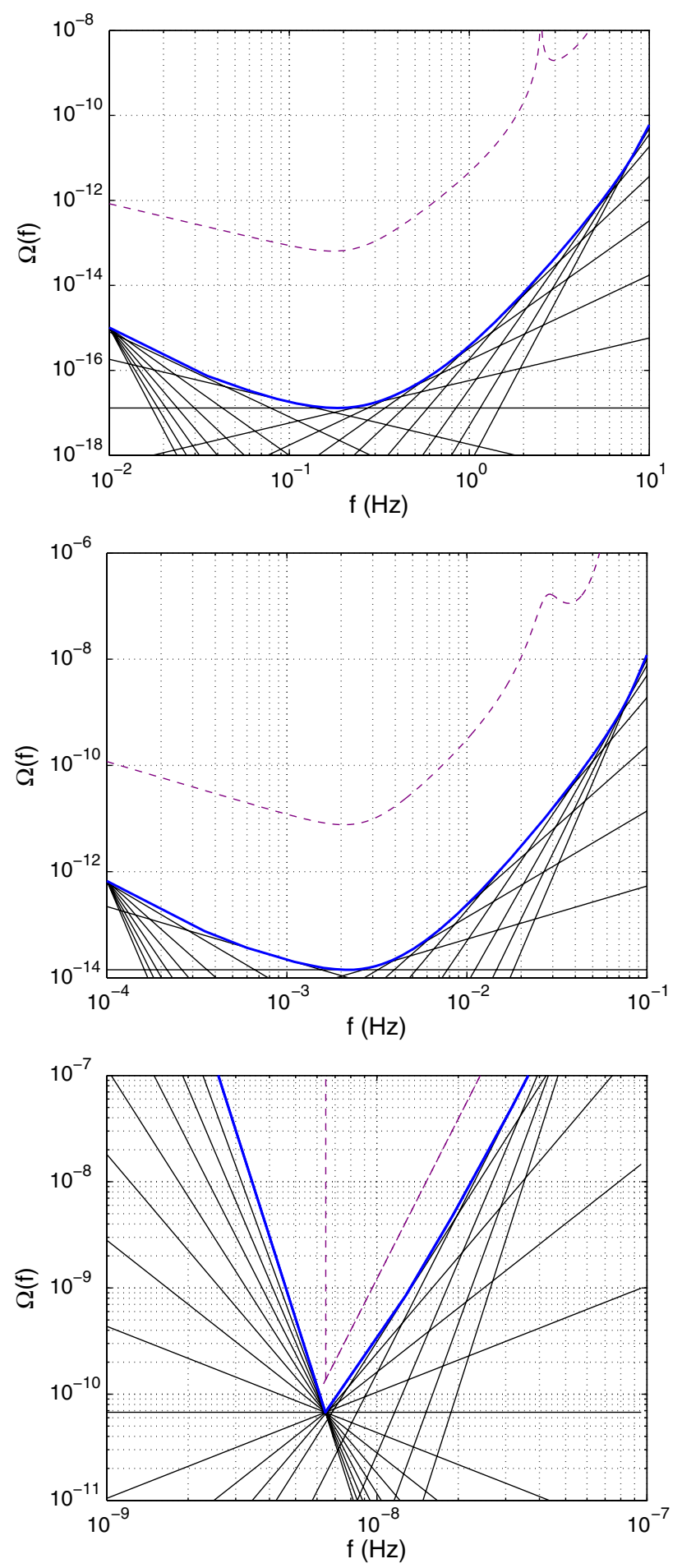

FIG. 10 (color online). One-sigma, power-law integrated sensitivity curves. The dashed purple curves show the effective strain spectral density $S_{\text {eff }}(f)$ [ $S_{n}(f)$ for LISA, middle panel] converted to fractional energy density units [see Eqs. (3), (23), and (27)]. Top panel: BBO assuming $T=1 \mathrm{yr}$ of observation. The spike at $\approx 2.5 \mathrm{~Hz}$ is due to a zero in the $\mathrm{BBO}$ overlap reduction function. Middle panel: LISA autocorrelation measurement assuming $T=1 \mathrm{yr}$ of observation and perfect subtraction of instrumental noise and/or any unwanted astrophysical foreground. Bottom panel: A pulsar timing array consisting of 20 pulsars, $100 \mathrm{~ns}$ timing noise, $T=5 \mathrm{yr}$ of observation, and a cadence of $20 \mathrm{yr}^{-1}$. 
where

$$
\begin{gathered}
(\widetilde{\delta x})^{2}=4 \times 10^{-22} \frac{\mathrm{m}^{2}}{\mathrm{~Hz}}, \\
(\widetilde{\delta a})^{2}=9 \times 10^{-30} \frac{\mathrm{m}^{2}}{\mathrm{~s}^{4} \cdot \mathrm{Hz}}
\end{gathered}
$$

are the position and acceleration noise $[6,11]$ and $L=5 \times$ $10^{9} \mathrm{~m}$ is the arm length. The transfer function $\mathcal{R}(f)$ is taken from Fig. 4, restricted to the LISA band, $10^{-4} \mathrm{~Hz}<f<$ $10^{-1} \mathrm{~Hz}$. Using the above expression for $\rho$ and following the same steps from the previous subsection for the construction of a power-law integrated curve, we obtain the sensitivity curve for LISA given in Fig. 10, middle panel.

Note that the minimum value of $\Omega(f)$ shown in this plot is about a factor of 10 times smaller than the value of $\Omega_{\mathrm{gw}}(f) \approx 2 \times 10^{-13}$ reported in [20,21]. Part of this difference is due to our use of $\rho=1$ for the sensitivity curve, while their value of $\Omega_{\mathrm{gw}}(f)$ corresponds to a strong (several $\sigma$ ) detection having a Bayes factor $\geq 30$. The remaining factor can probably be attributed to the marginalization over the instrumental noise and galactic foreground parameters in [20,21], while Eq. (36) assumes that we know these parameters perfectly.

\section{Pulsar timing array}

For the pulsar timing array sensitivity curve, we consider a network of 20 pulsars taken from the International Pulsar Timing Network (IPTA) [22], which we assume have identical white timing noise power spectral densities,

$$
P_{n}(f)=2 \Delta t \sigma^{2}
$$

where $1 / \Delta t$ is the cadence of the measurements, taken to be $20 \mathrm{yr}^{-1}$, and $\sigma$ is the root-mean-square timing noise, taken to be $100 \mathrm{~ns}$. We note that the pulsar timing network we envision may be somewhat optimistic, as $100 \mathrm{~ns}$ rootmean-square timing noise is ambitious. Also, we do not include the effects of fitting each pulsar's period $P$ and spin-down rate $\dot{P}$ to a timing model, which introduces both nonstationarity in the timing residuals and loss of sensitivity [23]. Nevertheless, one can still write down an analogous expression to Eq. (22) including these effects [24].

Since the timing noise power spectral densities are identical, it follows that

$$
S_{\text {eff }}(f)=S_{n}(f)\left[\sum_{I=1}^{M} \sum_{J>I}^{M} \zeta_{I J}^{2}\right]^{-1 / 2},
$$

where

$$
S_{n}(f)=P_{n}(f) / \mathcal{R}(f)=12 \pi^{2} f^{2} P_{n}(f)
$$

and $\zeta_{I J}$ are the Hellings and Downs factors for each pair of pulsars in the array. For our choice of 20 pulsars,

$$
\sum_{I=1}^{M} \sum_{J>I}^{M} \zeta_{I J}^{2}=4.74,
$$

which can thought of as the effective number of pulsar pairs for the network. Finally, we assume a total observation time $T=5 \mathrm{yr}$, which sets the lower frequency limit of $S_{\text {eff }}(f)$. Given these parameters, we expect the pulsar timing array to be operating in the "intermediate signal limit" [24]. We therefore utilize the scaling laws from Fig. 2 in Ref. [24] to adjust the power-law integrated curves, since Eqs. (21) and (22) for $\rho$ are valid in the weak-signal limit and overestimate the expected signal-to-noise ratio by a factor of $\approx 5$ for an observation of $T=5 \mathrm{yr}$. The power-law integrated curve for IPTA is given in Fig. 10, bottom panel.

It is interesting to note that the power-law integrated curves for Advanced LIGO and BBO are relatively round in shape, whereas the pulsar timing curve is pointy. [The steep $\Omega(f) \propto f^{5}$ spectrum can be understood as follows: The transfer function $\mathcal{R}(f)$ contributes a factor of $f^{2}$, while the conversion from power to energy density contributes an additional factor of $f^{3}$.] This reflects the fact that the sensitivity of pulsar timing measurements is mostly determined by a small band of the lowest frequencies regardless of the spectral shape of the signal. However, the timingmodel fit mentioned above may round out the pointy shape of the PTA sensitivity curve. We also note that the stochastic background in the PTA band may exhibit variability. The power-law integrated curves represent the sensitivity to energy density observed at the Earth over the course of the measurement.

Figure 11 is a summary of the results of this section, showing the power-law integrated sensitivity curves for the different detectors on a single plot spanning a wide range of frequencies.

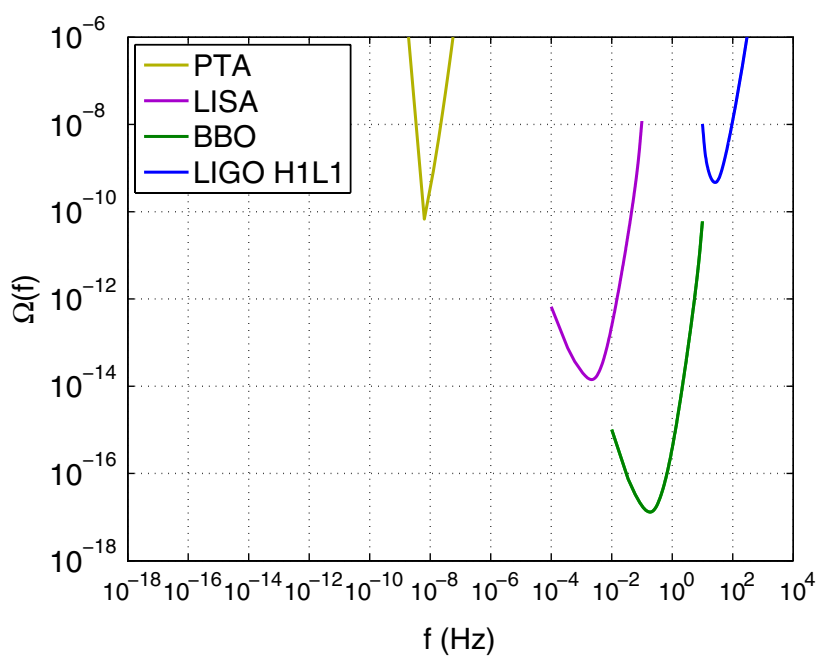

FIG. 11 (color online). One-sigma, power-law integrated sensitivity curves for the different detectors considered in this paper, plotted on the same graph. The Advanced LIGO H1L1, BBO, and pulsar timing sensitivity curves correspond to correlation measurements using two or more detectors. The LISA sensitivity curve corresponds to an autocorrelation measurement in a single detector assuming perfect subtraction of instrumental noise and/or any unwanted astrophysical foreground. 


\section{DISCUSSION}

We have presented a graphical representation of detector sensitivity curves for power-law gravitational-wave backgrounds that takes into account the enhancement in sensitivity that comes from integrating over frequency in addition to integrating over time. We applied this method to construct new power-law integrated sensitivity curves for crosscorrelation searches involving advanced ground-based detectors, BBO, and a network of pulsars from a pulsar timing array. We also constructed a power-law integrated sensitivity curve for an autocorrelation measurement using LISA. The new curves paint a more accurate picture of the expected sensitivity of upcoming observations. The code that we used to produce the new curves is available at https://dcc.ligo.org/ LIGO-P1300115/public for public download. Hopefully, this will allow other researchers to easily construct similar sensitivity curves. Required inputs are the noise power spectral density $P_{n I}(f)$ for each detector in the network and the overlap reduction function $\Gamma_{I J}(f)$ for each detector pair. Common default files are available for download with the plotting code.

Although the above discussion has focused on comparing predicted strengths of gravitational-wave backgrounds to sensitivity curves for current or planned detectors, one can also present measured upper limits for power-law backgrounds in a similar way. That is, instead of plotting the upper limits for $\Omega_{\beta}$ (for fixed $f_{\text {ref }}$ ) as a function of the spectral index $\beta$ as in $[2,25,26]$, one can plot the envelope of upper-limit power-law curves as a function of frequency. This would better illustrate the frequency dependence of the upper limits in the observing band of the detectors.

\section{ACKNOWLEDGMENTS}

We thank Vuk Mandic and Nelson Christensen for helpful comments regarding an earlier draft of the paper. J.D.R. would also like to thank Paul Demorest, Justin Ellis, Shane Larson, Alberto Sesana, and Alberto Vecchio for discussions related to PTA sensitivity curves. E. T. is a member of the LIGO Laboratory, supported by funding from the United States National Science Foundation. LIGO was constructed by the California Institute of Technology and Massachusetts Institute of Technology with funding from the National Science Foundation and operates under Cooperative Agreement No. PHY-0757058. J. D. R. acknowledges support from NSF Grants No. PHY-1205585, No. PHY-0855371, and No. CREST HRD-1242090.
[1] G. Hobbs, in High-Energy Emission from Pulsars and Their Systems, edited by D.F. Torres and N. Rea (Springer, Berlin-Heidelberg, 2011), Astrophysics and Space Science Proceedings, p. 229.

[2] B. Abbott et al., Nature (London) 460, 990 (2009).

[3] B. Allen and J. D. Romano, Phys. Rev. D 59, 102001 (1999).

[4] N. Christensen, Phys. Rev. D 46, 5250 (1992).

[5] É. É. Flanagan, Phys. Rev. D 48, 2389 (1993).

[6] N. J. Cornish and S. L. Larson, Classical Quantum Gravity 18, 3473 (2001).

[7] L. S. Finn, S. L. Larson, and J. D. Romano, Phys. Rev. D 79, 062003 (2009).

[8] R. W. Hellings and G. S. Downs, Astrophys. J. Lett. 265, L39 (1983).

[9] M. Anholm, S. Ballmer, J. D. E. Creighton, L. R. Price, and X. Siemens, Phys. Rev. D 79, 084030 (2009).

[10] S. Phinney et al., NASA Mission Concept Study (2004).

[11] J. Crowder and N. J. Cornish, Phys. Rev. D 72, 083005 (2005).

[12] C. Cutler and J. Harms, Phys. Rev. D 73, 042001 (2006).

[13] D. Shoemaker, Advanced LIGO Anticipated Sensitivity Curves (2010), https://dcc.ligo.org/LIGO-T0900288/public.
[14] C. Wu, V. Mandic, and T. Regimbau, Phys. Rev. D 85, 104024 (2012).

[15] J. Abadie et al., Classical Quantum Gravity 27, 173001 (2010).

[16] B. Iyer et al., LIGO-India Tech. Rep. 2011.

[17] C. Cutler, Phys. Rev. D 57, 7089 (1998).

[18] M. Tinto, J. W. Armstrong, and F. B. Estabrook, Phys. Rev. D 63, 021101 (2000).

[19] C. J. Hogan and P.L. Bender, Phys. Rev. D 64, 062002 (2001).

[20] M. R. Adams and N. J. Cornish, Phys. Rev. D 82, 022002 (2010).

[21] M. R. Adams and N. J. Cornish, arXiv:1307.4116.

[22] G. Hobbs et al., Classical Quantum Gravity 27, 084013 (2010).

[23] R. van Haasteren and Y. Levin, Mon. Not. R. Astron. Soc. 428, 1147 (2012).

[24] X. Siemens, J. Ellis, F. Jenet, and J. D. Romano, Classical Quantum Gravity 30, 224015 (2013).

[25] J. Abadie et al., Phys. Rev. D 85, 122001 (2012).

[26] V. Mandic, E. Thrane, S. Giampanis, and T. Regimbau, Phys. Rev. Lett. 109, 171102 (2012). 